\title{
VACCINATION IN PREGNANCY
}

\author{
SF NARGIS ${ }^{1}$, MS RAHMAN $^{2}$
}

\begin{abstract}
Routine vaccines that generally are safe to administer during pregnancy include diphtheria, tetanus, influenza, and hepatitis B, Other vaccines such as meningococcal and rabis, may be considered, Vaccines that are contraindicated because of the theoretic risk of fetal transmission include measles, mumps and rubella, varicella and bacilli Calmette-Guerin. A number of other vaccines have not yet been adequately studied; therefore risks of vaccination must be weighed against the risks of the disease to the mother and fetus. Inadvertent administration of any of these vaccination, however is not considered an indication for termination of the pregnancy.
\end{abstract}

\section{Introduction}

Immunization through vaccines is one of the most effective means of preventing disease, disability and death from infectious disease. Vaccination can be used during pregnancy, post partum and during breast feeding. Adult immunization rate have fallen partly because of misconceptions about the safety and benefits of current vaccines. The danger of these misconceptions is magnified during pregnancy when concerned physicians are hesitant to administer vaccines and patients are reluctant to accept them.

Many vaccine preventable diseases have serious consequences for the pregnant mother, the fetus, and the neonate. So vaccination included as a part of preconception care and provides recommendations for clinical care. Vaccinations that are recommended

1. Dr. Shamim Fatema Nargis, Assistant Professor Gynae and Obstetrics, SSMC and Mitford hospital, Dhaka.

2. Dr. Md. Shahinur Rahman, Assistant Professor Skin and VD., SSMC and Mitford hospital, Dhaka.

Correspondence to: Dr. Shamim Fatema Nargis, Assistant Professor Gynae and Obstetrics, SSMC and Mitford hospital, Dhaka. E-mail: nargisobgyn@yahoo.com highly in preconception care include the hepatitis B and the measles, mumps, and rubella vaccines. Human papilloma virus (HPV), varicella, diphtheria, tetanus, and pertussis vaccinations also has role as preconception care ${ }^{1}$.

Physicians should have the clear pictures about the vaccine which should given as preconception care, in pregnancy and in lactation, and if inadvertently given during pregnancy what happens. Physicians should consider vaccinating pregnant women on the basis of the risk of vaccination versus the benefit of protection in each particular situation, regardless of whether live or inactivated vaccine is used. Physicians should also reassure their patients that no vaccines are contraindicated during breastfeeding.

Vaccine commonly administered during pregnancy and their indications for use during pregnancy are summarized as, Immunization during pregnancy considered safe if other wise indicated are: -Tetanus and diphtheria toxoid (Td), Hepatitis B, Influenza. Immunization is contraindicated during pregnancy or safeties not established are: - BCG, Measeles, Mumps, Rubella, and Vericilla.

\section{The tetanus toxoid (TT)}

Tetanus and Diphtheria

The tetanus and diphtheria toxoids vaccine $(\mathrm{Td})$ is effective in preventing tetanus and diphtheria, two potentially life-threatening conditions. The tetanus toxoid (TT) alone, the tetanus toxoid and diphtheria (Td) toxoid is routinely recommended for susceptible pregnant women in second trimester of pregnancy. Pregnant women who are not immunized or partially immunized should complete the primary series ${ }^{2}$. Salama MM, Hady OA, Ashour W et al in their study 
conclude that the use of $\mathrm{Td}$ vaccine improves immunogenicity for both tetanus and diphtheria more than the use of TT vaccine alone ${ }^{3}$.

\section{The influenza vaccine}

The influenza vaccine should be administered to all pregnant women who will be in the second and third trimester of pregnancy during the influenza season. The influenza vaccine is a killed virus preparation with an annually adjusted antigenic make up. Immunization should be avoided in most patients during first trimester to avoid a coincidental association with spontaneous abortion which is common in the first trimester. However pregnant women with medical conditions that increase their risk for complications from influenza (e.g., asthema, cardiovascular disease, diabetes, suppressed immune system) should be vaccinated before the influenza season regardless of the pregnancy trimester.

\section{Hepatitis A and $B$ vaccination}

Safety of hepatitis A vaccination during pregnancy has not been determined. If a pregnant woman is exposed to hepatitis $A$, administration of immune globulin is strongly recommended ${ }^{2}$.

Vertical transmission is responsible for $35 \%-40 \%$ of the new cases of hepatitis B worldwide and it is associated with an increased risk of chronic hepatitis $B$, cirrhosis and hepatocellular carcinoma. Hepatitis $B$ vaccine contains noninfectious viral surface antigen particles and should cause no risk to the fetus; neither pregnancy nor lactation is a contraindication to vaccination ${ }^{2,4}$.

\section{Rubella or German measles}

Rubella or German measles is caused by the rubella virus. Although usually a benign infection in adults, rubella can result in birth defects that include cardiac, ophthalmologic, auditory and neurologic disorder. The measles, mumps and rubella vaccine (MMR) and its component vaccine should not be administered to pregnant women; women should counsel to avoid becoming pregnant within four weeks of vaccination. However, inadvertent vaccination of a pregnant woman is not considered to be a reason to terminate the pregnancy 2,5 . Preconception vaccination by MMR to the unexposed women protects rubella in pregnancy and its serious consequences ${ }^{6}$.

\section{Pertussis}

Severe pertussis disease in early infancy remains a significant problem, both in developed and under developed countries. Even intensive care support infants with pertussis pneumonia still die, so prevention is the key. The sources of pertussis in infants less than 3 months of age are usually adults. Because of high vaccination coverage among infants, adults and adolescents are now the main reservoir for Bordetella pertusis. Strategies to protect these infants may be indirect (timely primary immunization and boosters for older children and adults) and direct (mother during pregnancy or infant soon after birth). Adult booster immunization, either universal or targeted (mothers during pregnancy or 'cocoon'), would probably be effective but is challenging to implement. Vaccination of all adults with Tdap instead of dT vaccine is recommended in many countries, because this strategy may reduce the burden of disease in adults and infants and is cost effective ${ }^{7,8}$.

\section{Varicella}

Varicella -zoster virus causes chicken pox and may rarely cause serious complications such as encephalitis and pneumonia. The risk of these complications increases with age, and up to $15 \%$ of infected persons have herpes zoster later in life. The varicella vaccine contains live attenuated varicella zoater virus. Immunization during pregnancy is contraindicated. Women who are vaccinated should avoid becoming pregnant for one month following each injection. If susceptible pregnant woman is exposed to varicella however administration of varicella-zoster immunnoglobulin should be strongly considered. If a pregnant woman is inadvertently vaccinated with varicella vaccine or becomes pregnant within four weeks of being vaccinated she should be counseled regarding potential effects on the fetus. Theoretic risks to the fetus are very small, and exposure to the varicella vaccine is not an indication for termination of pregnancy ${ }^{2}$.

\section{Anthrax, Rabies, Polio}

Anthrax is caused by the spore-forming bacterium Bacillus antrasis, occurring in humans in three forms; cutaneous, inhalational and gastrointestinal. Anthrax vaccine usually not recommended in pregnancy, should be considered on a case by case basis ${ }^{2}$.

Rabies is a viral infection transmitted most commonly by the saliva of infected animals. Considering the 
potential maternal and fetal consequences of untreated rabies exposure similar guidelines should be used for post exposure prophylaxis in pregnant and non pregnant patients ${ }^{2}$.

Although no adverse effects have been documented with oral polio vaccine (OPV) or inactivated polio vaccine (IPV) in pregnant women or their fetuses, both vaccines should be avoided during pregnancy on a theoretic basis ${ }^{2}$.

\section{Pregnancy and traveling}

Many vaccinations are recommended for travelers, However live vaccines are contra indicated in pregnant women because of theoretical considerations. Exceptionally a yellow fever vaccination may be given after the first trimester. Killed, inactivated or polysaccharide vaccines can be given after the first trimester after a thorough risk/benefit evaluation. Because of the potentially devastation effect of malaria to the mother and the child, travelling to the endemic malaria regions should be avoided, chemoprophylaxis with mefloquine is indicated ${ }^{9}$.

\section{Typhoid vaccination}

The two types of typhoid vaccination is use today are live attenuated and oral vaccine and a parenteral polysaccharide vaccine, neither form of typhoid vaccine is officially recommended during pregnancy ${ }^{2}$.

Yellow fever and Japanese encephalitis (JE) vaccine

No specific evidence is available to demonstrate the safety of yellow fever immunization during pregnancy and Japanese encephalitis (JE) vaccine. Vaccination generally not recommended in pregnancy.

\section{BCG vaccine}

Use of BCG vaccine is not recommended during pregnancy as BCG immunization researches have not been studied extensively in pregnant women².

\section{HPV vaccine}

HPV vaccine efficacy studies for prevention of cervical cancer, and now also for prevention of vulval and vaginal cancers, confirmed $98-100 \%$ vaccine efficacy.

Pregnant women should not get the vaccine. Even though it appears to be safe for both mother and unborn baby, it is still being studied. If a women who is pregnant does get the vaccine this is not a reason to consider ending the pregnancy. Women who are breast feeding may safely get the vaccine ${ }^{10}$. Sjoborg KD and Eskild A in there study recommended that HPV $16 / 18$ vaccination programmes may reduce the number of preterm deliveries through reducing the need for cone excision ${ }^{11}$.

Infectious Diseases Committee of the Society of Obstetricians and Gynaecologists of Canada Recommendations 1. All women of childbearing age should be evaluated for the possibility of pregnancy before immunization. 2 . Health care providers should obtain an immunization history from all women accessing prenatal care. 3 . In general, live and/or liveattenuated virus vaccines are contraindicated during pregnancy, as there is a, largely theoretical, risk to the fetus. 4. Women who have inadvertently received immunization with live or live-attenuated vaccines during pregnancy should not be counseled to terminate the pregnancy because of a teratogenic risk. 5 . Nonpregnant women immunized with a live or liveattenuated vaccine should be counseled to delay pregnancy for at least four weeks. 6 . Inactivated viral vaccines, bacterial vaccines, and toxoids are considered safe in pregnancy. 7 . Women who are breastfeeding can still be immunized (passive-active immunization, live or killed vaccines). 8. Pregnant women should be offered the influenza vaccine when pregnant during the influenza season ${ }^{12}$.

\section{Conclusion:}

Immunization through vaccines is is one of the most effective means of preventing disease, disability and death from infectious disease. Health provider can play an important role in helping to prevent infectious disease during pregnancy and post partum by better understanding which vaccinations can be used in pregnancy and during breastfeeding. Implementation of the recommendations in the guideline globally should result in more appropriate immunization of pregnant and breastfeeding women, decreased risk of contraindicated immunization, and better disease prevention.

\section{References:}

1. Coonrod DV, Jack BW, Boggess KA, Long R, Conry JA, Cox SN, Cefalo R, Hunter KD, Pizzica $A$, Dunlop AL. The clinical content of preconception care: immunization as a part of preconception care. Am J Obstet Gynecol. 2008 Dec; 199(6 suppl 2):S290-5.

2. Denise K, Sur MD, David H, Wallis MD, Theodore $X, O$ Connell. Vaccinatioin in pregnancy. Am Fam Physician 2003; 68:E299-309. 
3. Salama MM, Hady OA, Ashour W, Mostafa A, El Alkamy S, El Sayed N, El Yazeed RA. A Randomizid controlled Tial of Oral Administration of Tetanus Toxoin (TT) Vursus Tetanus and Reduced Diptheria (Td) in Pregnant Women. J Clin Immunol.2009 Apr 14.

4. da Conceiao JS, Diniz-Santos DR, Ferreira CD, Paes FN,Melo CN, Silva LR. Knowledge of obstetricians about the vertical transmission of hepatitis B virus. Arq Gastroenterol. 2009 JanMar; 46 (1):57-61

5. Nasiri R, Yoseffi J, Khajedaloe M, Sarafraz Yazadi $M$, Delfoshaei F. Congenital rubella syndrome after rubella vaccination in 1-4 weeks periconceptional period. Indian J Pediatr. 2009 Mar; 76(3): 279-82.

6. Gupta SN, Gupta NN. An outbreak of rubella in a hilly district of Kangra-Chamba, Himachal Pradesh, India, 2006. Indian J Pediatr. 2009 Apr 16.

7. Mclntyre P, Wood N. Pertusis in early infancy: disease burden and preventive strategies.Curr Opin Infect Dis. 2009Jun;22 (3):215-23.
8. Riffelemann $\mathrm{M}$,Wirsing von Konig $\mathrm{CH}$. Life long protection against pertusis. Dtsch Med Wochenschr.2009 Apr; 134Suppl 2:S86-89. Epub2009 Apr 7.

9. Walentiny C. Pregnancy and traveling. Dtsch Med Wochen schr.2009 Mar; 134(12): 594-8.Epub 2009 Mar 10.

10. Jenson HB. Human papillomavirus vaccub: a paradigm shift for pediatricians. Curr Opin Pediatr. 2009 Feb; 21(1): 112-21.

11. Sjoborg KD and Eskild $A$. Vaccination against human papilloma virus-an impact on pretermdelivery? Estimations based on literature review. Acta obstet GynecolScand.2009; 88(3) 255-60

12. Gruslin A, Steben M, Halperin S, Money DM, Yudin MH, Boucher M, Cormier B, Oglivie G, Paquet C, Steenbeek A, Van Eyk N, Van Schlkwyk J, Wong T. Immunization in pregnancy. J Obstet Gynaecol Can. 2008 Dec; 30(12):114954. 